\title{
ASYMPTOTIC STABILITY OF THE WAVE EQUATION ON COMPACT MANIFOLDS AND LOCALLY DISTRIBUTED VISCOELASTIC DISSIPATION
}

\author{
MARCELO M. CAVALCANTI, VALÉRIA N. DOMINGOS CAVALCANTI, \\ AND FLÁVIO A. F. NASCIMENTO \\ (Communicated by James E. Colliander)
}

\begin{abstract}
We discuss the asymptotic stability of the wave equation on a compact Riemannian manifold $(M, \mathbf{g})$ subject to locally distributed viscoelastic effects on a subset $\omega \subset M$. Assuming that the well-known geometric control condition $\left(\omega, T_{0}\right)$ holds and supposing that the relaxation function is bounded by a function that decays exponentially to zero, we show that the solutions of the corresponding partial viscoelastic model decay exponentially to zero. We give a new geometric proof extending the prior results in the literature from the Euclidean setting to compact Riemannian manifolds (with or without boundary).
\end{abstract}

\section{INTRODUCTION}

1.1. Results and methodology. Let $(M, \mathbf{g})$ be an $n$-dimensional compact Riemannian manifold with boundary where $\mathbf{g}$ denotes a Riemannian metric of class $C^{\infty}$. We denote by $\nabla$ the Levi-Civita connection on $M$ and by $\Delta$ the LaplaceBeltrami operator on $M$.

In this paper, we investigate the stability properties of function $u(x, t)$ which solve the following partially viscoelastic problem:

$$
\begin{cases}u_{t t}-\kappa_{0} \Delta u+\int_{0}^{t} g(t-s) \operatorname{div}[a(x) \nabla u(s)] d s=0 & \text { on } M \times] 0, \infty[, \\ u=0 & \text { on } \partial M \times] 0, \infty[, \\ u(0)=u^{0}, \quad u_{t}(0)=u^{1}, & x \in M,\end{cases}
$$

where $\kappa_{0}>0, g$ is the relaxation function and $a(x) \geq a_{0}>0$ in a subset $\omega \subset M$. Assuming that the well-known geometric control condition $\left(\omega, T_{0}\right)$ holds and supposing that the kernel of the memory term $g$ is dominated by a function which decays exponentially to zero, we show that the solutions to the corresponding partial viscoelastic model decay exponentially to zero no matter how small the viscoelastic portion of the material. We give a new geometric proof of a result due to Muñoz Rivera and Salvatierra [RI-SA] using microlocal analysis, which allows us

Received by the editors November 28, 2011.

2010 Mathematics Subject Classification. Primary 35L05, 34Dxx, 35A27.

Key words and phrases. Wave equation, compact Riemannian manifold, viscoelastic distributed damping.

Research of the first author was partially supported by the CNPq Grant 300631/2003-0.

Research of the second author was partially supported by the CNPq Grant 304895/2003-2.

The third author, a doctorate student at the State University of Maringá, was partially supported by a grant of CNPq, Brazil. 
not only to improve the assumptions imposed on the relaxation function but also to extend the result from the Euclidean setting to a compact Riemannian manifold (with or without boundary). Regarding asymptotic stability, the majority of works in the previous literature in connection with viscoelastic problems make use of the multiplier method combined with a Liapunov functional suitably chosen. In the present paper we base the proof of exponential stability to problem (1.1) on arguments of contradiction as considered, for instance, in DEH-LE-ZUA. In addition, to obtain the so-called contradiction, it is essential to use a unique continuation property. Although in the Euclidean setting Holmgren's uniqueness theorem is enough, on Riemannian compact manifolds we need to employ the inverse observability property introduced in BAR-LE-RAU]. Indeed, we are assuming that the geometric control condition holds, namely, that the geodesics of $\bar{M}$ have no contact of infinite order with $\partial M$ and that there exists $T_{0}>0$ such that every geodesic travelling at speed 1 and issued at $t=0$ meets $\bar{\omega}$ in a time $t<T_{0}$. Then, we guarantee the existence of a constant $C=C\left(T_{0}, \omega\right)>0$ verifying

$$
E_{u}(0) \leq C \int_{0}^{T_{0}} \int_{\omega}\left|u_{t}(x, t)\right|^{2} d M d t
$$

for every solution $u$ of

$$
\left\{\begin{array}{l}
u_{t t}-\Delta u=0 \text { in } M \times(0, \infty), \\
u=0 \quad \text { in } \partial M \times(0, \infty), \\
\left(u(0), u_{t}(0)\right)=\left(u_{0}, u_{1}\right),
\end{array}\right.
$$

with initial data $\left(u_{0}, u_{1}\right) \in H_{0}^{1}(M) \times L^{2}(M)$ and $E_{u}(t)=\frac{1}{2} \int_{M}\left[\left|u(x, t)^{2}\right|+|\nabla u|^{2}\right] d M$. The unique continuation property given in (1.2) and new tools closely related to problem (1.1) are the main ingredients to establish the desired exponential stability.

1.2. Literature overview. In what concerns the Euclidean setting, the linear or semilinear wave equation subject to locally distributed frictional damping, we can mention the classical works [DEH-LE-ZUA], [MAR, [NA], [NA1, [TOUN], ZUA]. Regarding the propagation of the wave equation on compact manifolds, it is worth mentioning the following papers: BAR-LE-RAU, BEL, CA-DO-FU-SO-2, CA-DO-FU-SO, CA-DO-FU-SO-1, [CHR, HI], LE, [MI, DA-LA-TO, [RAU-TAY], TRI-YAO]. On the other hand, there is a huge number of works published concerning the viscoelastic wave equation in the Euclidean setting. However, very few are related to locally distributed viscoelastic effects, as, for example, CA-OQ, GES-MES and RI-SA, but even so they consider the viscoelastic wave equation in the Euclidean setting. To the best of our knowledge the present paper is one of the pioneers in studying the asymptotic behaviour of the wave equation subject to locally distributed viscoelastic effects on compact Riemannian manifolds.

We would like to emphasize that the approach introduced in the present paper allows us to treat the linear wave equation subject to nonlinear locally distributed viscoelastic effects, namely,

$$
u_{t t}-\Delta u+g * \operatorname{div}(\sigma(a(x) \nabla u))=0,
$$

where the function $\sigma(\cdot)$ possesses suitable properties (see, for instance, the paper due to Qin Tiehu QIN and the references therein). However, in order to make our proof simpler, we shall focus our attention on the linear case. On the other hand, 
an interesting open problem is to investigate the semilinear wave equation,

$$
u_{t t}-\Delta u+f(u)+g * \operatorname{div}(a(x) \nabla u)=0,
$$

even if we consider a linear viscoelastic effect. In this case, we conjecture that the techniques used in [DEH-LE-ZUA], developed by Gerárd [GE] (see also Lebeau $[\mathrm{LE})$ and using microlocal defect measure (m.d.m.), can work, at least if the semilinear function $f(\cdot)$ has a sub-critical growth.

Our paper is organized as follows. Section 2 is concerned with the assumptions and the main result. We also introduce some notation. Section 3 is devoted to the proof of the main theorem.

\section{Assumptions And the main RESUlt}

The following assumptions are required:

Assumption 2.1. The relaxation function $g:\left[0, \infty\left[\rightarrow \mathbb{R}_{+}\right.\right.$is a $C^{1}$ nonincreasing function and satisfies

$$
\|a\|_{L^{\infty}} \int_{0}^{\infty} g(s) d s<\kappa_{0} .
$$

In addition, let us assume that

$$
g^{\prime}(t) \leq-\xi g(t), \text { for all } t \geq 0,
$$

for some $\xi>0$.

To obtain the stabilization of problem (1.1), we shall need the following geometrical assumption:

Assumption 2.2 (Geometric control condition). If $M$ is a manifold with boundary, we assume that the geodesics of $\bar{M}$ have no contact of infinite order with $\partial M$. Let $\omega^{\prime}$ be an open subset of $M$ and consider that there exists $T_{0}>0$ such that every geodesic travelling at speed 1 and issued at $t=0$ meets $\overline{\omega^{\prime}}$ in a time $t<T_{0}$.

We also assume that $a \in C^{\infty}(M)$ is a nonnegative function such that

$$
a(x) \geq a_{0}>0 \quad \text { in } \omega,
$$

where $\omega$ is an open subset verifying $\omega \supset \overline{\omega^{\prime}}$.

If $\partial M \neq \emptyset$ we define $\left.\Sigma_{T}=M \times\right] 0, T[$ and we set

$$
H_{0}^{1}(M):=\left\{v \in H^{1}(M) ;\left.v\right|_{\partial M}=0\right\},
$$

which is a Hilbert space with the topology endowed by $H^{1}(M)$.

The condition $\left.v\right|_{\partial M}=0$ is required to guarantee the Poincaré inequality,

$$
\|h\|_{L^{2}(M)}^{2} \leq\left(\lambda_{1}\right)^{-1}\|\nabla h\|_{L^{2}(M)}^{2}, \quad \text { for all } h \in H_{0}^{1}(M),
$$

where $\lambda_{1}$ is the first eigenvalue of the Laplace-Beltrami operator for the Dirichlet problem. If $\partial M=\emptyset$, see Remark 2.1. Using the standard Faedo-Galerkin method we obtain the following result:

Theorem 2.1. If $\left(u^{0}, u^{1}\right) \in\left[H^{2}(M) \cap H_{0}^{1}(M)\right] \times H_{0}^{1}(M)$ there exists a unique regular solution to problem (1.1) in the class

$u \in L_{l o c}^{\infty}\left(0, \infty ; H_{0}^{1}(M) \cap H^{2}(M)\right), u_{t} \in L_{l o c}^{\infty}\left(0, \infty ; H_{0}^{1}(M)\right), u_{t t} \in L_{l o c}^{\infty}\left(0, \infty ; L^{2}(M)\right)$. 
If $\left(u^{0}, u^{1}\right) \in H_{0}^{1}(M) \times L^{2}(M)$ and considering standard arguments of density, we prove that problem (1.1) has a unique weak solution in the class

$$
u \in C^{0}\left([0, \infty) ; H_{0}^{1}(M)\right) \cap C^{1}\left([0, \infty) ; L^{2}(M)\right) .
$$

To simplify the notation we introduce the binary operators

$$
\begin{aligned}
(g * w)(t) & :=\int_{0}^{t} g(t-s) w(s) d s, \\
(g \square w)(t) & :=\int_{0}^{t} g(t-s)|w(t)-w(s)|^{2} d s .
\end{aligned}
$$

The following lemma establishes an important relationship between these operators.

Lemma 2.2. For any $g, w \in C^{1}(\mathbb{R})$ we obtain the equality

$$
2[g * w] w^{\prime}=g^{\prime} \square w-g(t)|w|^{2}-\frac{d}{d t}\left\{g \square w-\left(\int_{0}^{t} g\right)|w|^{2}\right\} .
$$

Proof. Differentiating the expression

$$
g \square w-\left(\int_{0}^{t} g(s) d s\right)|w|^{2},
$$

our conclusion follows.

Assuming that $u$ is the unique global weak solution to problem (1.1), we define the corresponding energy functional by

$$
E_{u}(t)=\frac{1}{2} \int_{M}\left[\left|u_{t}(x, t)\right|^{2}+\kappa(x, t)|\nabla u(x, t)|^{2}+a(x) g \square \nabla u\right] d M,
$$

where

$$
\kappa(x, t):=\kappa_{0}-a(x) \int_{0}^{t} g(s) d s .
$$

Note that, in view of (2.1), we have that

$$
0<\kappa_{0}-\|a\|_{L^{\infty}} \int_{0}^{\infty} g(s) d s \leq \kappa(x, t) \leq \kappa_{0}, \quad \forall(x, t) \in M \times \mathbb{R}_{+} .
$$

The first order energy satisfies the following identity:

\section{Lemma 2.3.}

$$
\frac{d}{d t} E_{u}(t)=\frac{1}{2} \int_{M} a(x)\left[g^{\prime} \square \nabla u-g(t)|\nabla u|^{2}\right] d M .
$$

Proof. Multiplying equation (1.1) by $u_{t}$, performing integration by parts and using Lemma 2.2 we obtain the desired result.

As a consequence of Lemma 2.3. every solution of (1.1) in the class (2.6) satisfies the following identity:

$$
E_{u}\left(t_{2}\right)-E_{u}\left(t_{1}\right)=\frac{1}{2} \int_{t_{1}}^{t_{2}} \int_{M} a(x)\left[g^{\prime} \square \nabla u-g(t)|\nabla u|^{2}\right] d M d t, \text { for all } t_{2}>t_{1} \geq 0,
$$

and therefore the energy is a nonincreasing function of the time variable $t$. 
Theorem 2.4. Let us assume that assumptions (2.1) and (2.2) are in place. Then, for all $L>0$, there exist positive constants $C_{0}, \gamma$ (which depend on $L$ ) such that every weak solution of problem (1.1) satisfies

$$
E_{u}(t) \leq C_{0} e^{-\gamma t} E_{u}(0), \text { for all } t \geq T_{0},
$$

provided that $\left(u_{0}, u_{1}\right) \in H_{0}^{1}(M) \times L^{2}(M)$ and $E_{u}(0) \leq L$, where $E_{u}(t)$ is given in (2.7).

Remark 2.1. If $M$ is a compact Riemannian manifold without boundary, problem (1.1) is replaced by

$$
\left\{\begin{array}{l}
u_{t t}-\Delta u+u+g *[\operatorname{div}(a(x) \nabla u)]=0 \text { in } M \times(0, \infty), \\
\left(u(0), u_{t}(0)\right)=\left(u_{0}, u_{1}\right),
\end{array}\right.
$$

to obtain the $H^{1}(M)$ topology.

Under the same assumptions (2.1) and (2.2), the result of Theorem 2.4 remains true for initial data $\left(u_{0}, u_{1}\right) \in H^{1}(M) \times L^{2}(M)$. In this case the energy is defined by

$$
E_{u}(t)=\frac{1}{2} \int_{M}\left[\left|u_{t}(x, t)\right|^{2}+\kappa(x, t)|\nabla u(x, t)|^{2}+|u(x, t)|^{2}+a(x) g \square \nabla u\right] d M .
$$

\section{Proof of Theorem 2.4}

Our aim is to prove that the inequality

$$
E_{u}(T) \leq C\left\{\int_{0}^{T} \int_{M} a(x)\left[-g^{\prime} \square \nabla u+g(t)|\nabla u|^{2}\right] d M d t\right\}
$$

holds for all $T>T_{0}$, provided the initial $\left(u_{0}, u_{1}\right)$ data is taken in bounded sets of $H_{0}^{1}(M) \times L^{2}(M)$. Since $E_{u}(t)$ is a nonincreasing function, according to (2.9), it is enough to prove the following result:

Lemma 3.1. Let us assume hypotheses (2.1) and (2.2). Then, for all $T>T_{0}$ and for all $L>0$, there exists a positive constant $C=C(T, L)$ such that the inequality

$$
E_{u}(0) \leq C\left\{\int_{0}^{T} \int_{M} a(x)\left[-g^{\prime} \square \nabla u+g(t)|\nabla u|^{2}\right] d M d t\right\}
$$

holds for every solution $u$ of problem (1.1) provided that the initial data satisfies

$$
E_{u}(0) \leq L \text {. }
$$

Proof. We argue by contradiction. For simplicity we shall denote $u^{\prime}:=u_{t}$. Suppose that (3.2) is not verified and let $\left\{u_{k}(0), u_{k}^{\prime}(0)\right\}$ be a sequence of initial data where the corresponding solutions $\left\{u_{k}\right\}_{k \in \mathbb{N}}$ of (1.1) with $E_{u_{k}}(0)$, assumed uniformly bounded in $k$, verifies

$$
\lim _{k \rightarrow+\infty} \frac{E_{u_{k}}(0)}{\int_{0}^{T} \int_{M} a(x)\left[-g^{\prime} \square \nabla u_{k}+g(t)\left|\nabla u_{k}\right|^{2}\right] d M d t}=+\infty
$$

that is,

$$
\lim _{k \rightarrow+\infty} \frac{\int_{0}^{T} \int_{M} a(x)\left[-g^{\prime} \square \nabla u_{k}+g(t)\left|\nabla u_{k}\right|^{2}\right] d M d t}{E_{u_{k}}(0)}=0 .
$$


Since $E_{u_{k}}(t) \leq E_{u_{k}}(0) \leq L$, where $L$ is a positive constant, we obtain a subsequence, still denoted by $\left\{u_{k}\right\}$, which verifies the convergence

$$
\begin{aligned}
& u_{k} \rightarrow u \text { weakly in } H^{1}(M \times(0, T)), \\
& u_{k} \rightarrow u \text { weak star in } L^{\infty}\left(0, T ; H_{0}^{1}(M)\right), \\
& u_{k}^{\prime} \rightarrow u^{\prime} \text { weak star in } L^{\infty}\left(0, T ; L^{2}(M)\right) .
\end{aligned}
$$

Employing compactness results we also deduce that

$$
u_{k} \rightarrow u \text { strongly in } L^{2}\left(0, T ; L^{2}(M)\right) \text {. }
$$

At this point we will divide our proof into two cases, namely, $u \neq 0$ and $u=0$. Case (I) $u \neq 0$.

We observe that from (3.5) and having in mind that $E_{u_{k}}(0) \leq L$ for all $k \in \mathbb{N}$, we have

$$
\lim _{k \rightarrow+\infty} \int_{0}^{T} \int_{M} a(x)\left[-g^{\prime} \square \nabla u_{k}+g(t)\left|\nabla u_{k}\right|^{2}\right] d M d t=0 .
$$

From (3.10) we deduce that

$g(t) a(x)\left|\nabla u_{k}\right|^{2}+\int_{0}^{t}-g^{\prime}(t-s) a(\cdot)\left|\nabla u_{k}(\cdot, t)-\nabla u_{k}(\cdot, s)\right|^{2} d s \rightarrow 0$ in $L^{1}(M \times(0, T))$.

On the other hand, since $a(x) \geq a_{0}>0$ in $\omega$ and $g(t) \geq g(T)$ for all $t \in[0, T]$, we infer that

$$
\begin{aligned}
& \int_{0}^{T} \int_{M} a(x) g(t)\left|\nabla u_{k}\right|^{2} d M d t \\
& =\int_{0}^{T} \int_{M \backslash \omega} a(x) g(t)\left|\nabla u_{k}\right|^{2} d M d t+\int_{0}^{T} \int_{\omega} a(x) g(t)\left|\nabla u_{k}\right|^{2} d M d t \\
& \geq \int_{0}^{T} \int_{\omega} a(x) g(t)\left|\nabla u_{k}\right|^{2} d M d t \geq a_{0} g(T) \int_{0}^{T} \int_{\omega}\left|\nabla u_{k}\right|^{2} d M d t .
\end{aligned}
$$

The last inequality and (3.11) yields

$$
\lim _{k \rightarrow+\infty} \int_{0}^{T} \int_{\omega}\left|\nabla u_{k}\right|^{2} d M d t=0 .
$$

Combining (3.9) and (3.13) we deduce that $\nabla u=0$ in $L^{2}\left(0, T ; L^{2}(\omega)\right)$, and, consequently, $u(x, t)=C(t)$ a.e. in $\omega \times(0, T)$. Since $u(t)=0$ a.e. on $\partial M$, it holds that $C(t)=0$ a.e. in $(0, T)$, which implies that $u=0$ a.e. in $\omega \times(0, T)$. Passing to the limit in the equation, $u_{k}^{\prime \prime}-\Delta u_{k}+\operatorname{div}\left[a(x) g * \nabla u_{k}\right] d s=0$ in $M \times(0, T)$ when $k \rightarrow+\infty$, we get, taking (2.2) and (3.11) into account, that

$$
\begin{cases}u_{t t}-\Delta u & =0 \text { in } M \times(0, T) \\ u & =0 \text { in } \omega \times(0, T)\end{cases}
$$

Remark 3.1. (a) It should be possible to obtain $u=0$ a.e. in $\omega \times(0, T)$ directly from the Poincaré inequality.

(b) At this point we need to make use of a unique continuation result for the linear equation (3.14). Indeed, according to assumption (2.2), we are assuming that the geodesics of $\bar{M}$ have no contact of infinite order with $\partial M$ and, in addition, that there exists $T_{0}>0$ such that every geodesic traveling at speed 1 and issued at $t=0$ 
meets $\overline{\omega^{\prime}}$ in a time $t<T_{0}$. Then there exists a constant $C=C\left(T_{0}, \omega^{\prime}\right)>0$ such that

$$
E_{u}(0) \leq C \int_{0}^{T_{0}} \int_{\omega^{\prime}}\left|u_{t}(x, t)\right|^{2} d M d t
$$

holds for every solution $u$ of

$$
\left\{\begin{array}{l}
u_{t t}-\Delta u=0 \text { in } M \times(0, \infty), \\
u=0 \quad \text { in } \partial M \times(0, \infty), \\
\left.\left(u(0), u_{t} 0\right)\right)=\left(u_{0}, u_{1}\right),
\end{array}\right.
$$

with initial data $\left(u_{0}, u_{1}\right) \in H_{0}^{1}(M) \times L^{2}(M)$. Note that (3.15) implies that whenever two weak solutions are locally close to each other they must be globally close. In particular, two solutions that coincide in $\omega^{\prime} \times\left(0, T_{0}\right)$ must be identical. It was proved in BAR-LE-RAU that a necessary and sufficient condition to obtain inequality (3.15) is precisely the geometric control condition given in assumption (2.2). We recall that we are assuming that $\omega \supset \overline{\omega^{\prime}}$ and $a(x) \geq a_{0}>0$ in $\omega$.

Since $u=0$ a.e. in $\omega \times(0, T), u_{t}=0$ a.e. in $\omega \times(0, T)$ and $T>T_{0}$, from the uniqueness result given by Remark 3.1 we conclude that $u \equiv 0$, which is a contradiction.

Case (II) $u=0$.

Defining

$$
c_{k}:=\left[E_{u_{k}}(0)\right]^{1 / 2}
$$

and

$$
\bar{u}_{k}:=\frac{1}{c_{k}} u_{k},
$$

and setting

$$
E_{\bar{u}_{k}}(t):=\frac{1}{2} \int_{M}\left|\bar{u}_{k}^{\prime}\right|^{2} d M+\frac{1}{2} \kappa(x, t) \int_{M}\left|\nabla \bar{u}_{k}\right|^{2} d M+\frac{1}{2} \int_{M} a(x)\left(g \square \nabla \bar{u}_{k}\right)(t) d M,
$$

we deduce that

$$
\begin{aligned}
E_{\bar{u}_{k}}(0): & =\frac{1}{2} \int_{M}\left|\bar{u}_{k}^{\prime}(0)\right|^{2} d M+\frac{1}{2} \int_{M}\left|\nabla \bar{u}_{k}(0)\right|^{2} d M \\
& =\frac{1}{2} \int_{M} \frac{\left|u_{k}^{\prime}(0)\right|^{2}}{c_{k}^{2}} d M d t+\frac{1}{2} \int_{M} \frac{\left|\nabla u_{k}(0)\right|^{2}}{c_{k}^{2}} d M=\frac{1}{E_{u_{k}}(0)} E_{u_{k}}(0)=1 .
\end{aligned}
$$

For a subsequence $\left\{\bar{u}_{k}\right\}$, we obtain

$$
\begin{aligned}
& \bar{u}_{k} \rightarrow \bar{u} \text { weak star in } L^{\infty}\left(0, T ; H_{0}^{1}(M)\right), \\
& \bar{u}_{k}^{\prime} \rightarrow \bar{u}^{\prime} \text { weak star in } L^{\infty}\left(0, T ; L^{2}(M)\right), \\
& \bar{u}_{k} \rightarrow \bar{u} \text { strongly in } L^{2}\left(0, T ; L^{2}(M)\right) .
\end{aligned}
$$

We observe that from (3.5) we can deduce that

$g(t) a(x)\left|\nabla \bar{u}_{k}\right|^{2}+\int_{0}^{t}-g^{\prime}(t-s) a(\cdot)\left|\nabla \bar{u}_{k}(\cdot, t)-\nabla \bar{u}_{k}(\cdot, s)\right|^{2} d s \rightarrow 0$ in $L^{1}(M \times(0, T))$. 
From (3.5) and (3.12) we also deduce that

$$
\lim _{k \rightarrow+\infty} \int_{0}^{T} \int_{\omega}\left|\nabla \bar{u}_{k}\right|^{2} d M d t=0 .
$$

In addition, $\bar{u}_{k}$ satisfies the equation

$$
\bar{u}_{k}^{\prime \prime}-\Delta \bar{u}_{k}+\operatorname{div}\left[a(x) g * \nabla \bar{u}_{k}\right]=0 \quad \text { in } M \times(0, T) .
$$

Passing to the limit in (3.25) when $k \rightarrow+\infty$ and taking (2.2) and (3.23) into account, we obtain, as considered in part (i), that

$$
\left\{\begin{aligned}
\bar{u}^{\prime \prime}-\Delta \bar{u} & =0 & & \text { on } M \times(0, T), \\
\bar{u} & =0 & & \text { on } \omega \times(0, T) .
\end{aligned}\right.
$$

Applying the same idea used in case (I) we have that $\bar{u}_{t}=0$ a.e. in $\omega \times(0, T)$, which implies that $\bar{u}=0$. Using the identity of the energy

$$
E_{\bar{u}_{k}}(T)=E_{\bar{u}_{k}}(0)+\frac{1}{2} \int_{0}^{T} \int_{M} a(x)\left[g^{\prime} \square \nabla \bar{u}_{k}-g(t)\left|\nabla \bar{u}_{k}\right|^{2}\right] d M d t
$$

and considering (3.19), (3.21) and (3.23), we deduce that

$$
\lim _{k \rightarrow+\infty} E_{\bar{u}_{k}}(t)=1, \text { for all } t \in[0, T] .
$$

Let $\varepsilon>0$ be small enough such that $T-\varepsilon>T_{0}$ and let us consider a cutoff function $\theta \in C_{0}^{\infty}(0, T)$ such that $\theta=1$ in $[\varepsilon, T-\varepsilon], 0 \leq \theta \leq 1$. In addition, consider another cutoff function $\varphi \in C^{\infty}(M)$ such that $\varphi=1$ in $\overline{\omega^{\prime}} \Subset \omega, \varphi=0$ in $M \backslash \omega$, $0 \leq \varphi \leq 1$. Returning to (3.25) we obtain

$$
\begin{aligned}
& -\int_{0}^{T} \int_{M} \bar{u}_{k}^{\prime} \bar{u}_{k} \varphi \theta^{\prime} d M d t-\int_{0}^{T} \int_{M}\left|\bar{u}_{k}^{\prime}\right|^{2} \theta \varphi d M d t+\int_{0}^{T} \int_{M}\left|\nabla \bar{u}_{k}\right|^{2} \theta \varphi d M d t \\
& +\int_{0}^{t} \int_{M}\left(\nabla \bar{u}_{k} \cdot \nabla \varphi\right) \bar{u}_{k} \theta d M d t+\int_{0}^{T} \int_{M} \operatorname{div}\left[a(x) g * \nabla \bar{u}_{k}\right] \bar{u}_{k} \varphi \theta d M d t=0 .
\end{aligned}
$$

Having in mind that $\bar{u} \equiv 0$, by combining (3.20) - (3.23) and (3.28), we deduce that

$$
\lim _{k \rightarrow+\infty}\left[-\int_{0}^{T} \int_{M}\left|\bar{u}_{k}^{\prime}\right|^{2} \theta \varphi d M d t+\int_{0}^{T} \int_{M}\left|\nabla \bar{u}_{k}\right|^{2} \theta \varphi d M d t\right]=0,
$$

and from the properties of the function $\varphi$, we obtain that

$$
\lim _{k \rightarrow+\infty}\left[-\int_{0}^{T} \int_{\omega}\left|\bar{u}_{k}^{\prime}\right|^{2} \theta \varphi d M d t+\int_{0}^{T} \int_{\omega}\left|\nabla \bar{u}_{k}\right|^{2} \theta \varphi d M d t\right]=0 .
$$

The last convergence combined with (3.24) yields

$$
\lim _{k \rightarrow+\infty} \int_{0}^{T} \int_{\omega}\left|\bar{u}_{k}^{\prime}\right|^{2} \theta \varphi d M d t=0 .
$$

From the above we deduce that

$$
\lim _{k \rightarrow+\infty} \int_{\varepsilon}^{T-\varepsilon} \int_{\omega^{\prime}}\left|\bar{u}_{k}^{\prime}\right|^{2} d M d t=0 \text { and } \lim _{k \rightarrow+\infty} \int_{\varepsilon}^{T-\varepsilon} \int_{\omega^{\prime}}\left|\nabla \bar{u}_{k}\right|^{2} d M d t=0
$$


Note that from Assumption 2.2 taking (3.29) into account and observing that $E_{\bar{u}_{k}}(t) \leq E_{\bar{u}_{k}}(0)=1$ for all $t \in[0, T]$ and $k \in \mathbb{N}$, we have, as in (3.15),

$$
1=E_{\bar{u}_{k}}(0) \leq C_{T_{0}, \omega^{\prime}, \varepsilon} \int_{\varepsilon}^{T-\varepsilon} \int_{\omega^{\prime}}\left|\bar{u}_{k}^{\prime}(x, t)\right|^{2} d M d t \rightarrow 0 \text { as } k \rightarrow \infty,
$$

which is a contradiction. The lemma is proved.

From Lemma 3.1 and from the fact that $E_{u}(t)$ is a nonincreasing function, (3.1) is in place. On the other hand, from (2.9)

$$
E_{u}(T)-E_{u}(0)=\frac{1}{2} \int_{0}^{T} \int_{M} a(x)\left[g^{\prime} \square \nabla u-g(t)|\nabla u|^{2}\right] d M d t .
$$

Then,

$$
E_{u}(T) \leq-C\left[E_{u}(T)-E_{u}(0)\right], \text { for all } T>T_{0} .
$$

From now on we proceed verbatim as in Muñoz-Rivera and Salvatierra RI-SA]. From (3.30) we infer that

$$
E_{u}(T) \leq \frac{C}{1+C} E_{u}(0)=\frac{1}{1+\frac{1}{C}} E_{u}(0), \text { for all } T>T_{0} .
$$

Repeating the above process from $T$ to $2 T$ we obtain

$$
E_{u}(2 T) \leq \frac{1}{1+\frac{1}{C}} E_{u}(T) \leq \frac{1}{\left(1+\frac{1}{C}\right)^{2}} E_{u}(0) .
$$

In general, we have that

$$
E_{u}(n T) \leq \frac{1}{\left(1+\frac{1}{C}\right)^{n}} E_{u}(0)
$$

Since any number $t$ can be written as $t=n T+r$ where $0 \leq r<T$ and $E_{u}(t)$ is a decreasing function, one has

$$
E(t) \leq E(t-r) \leq \frac{1}{\left(1+\frac{1}{C}\right)^{\frac{t-r}{T}}} E_{u}(0)=C_{0} e^{-\gamma t} E_{u}(0),
$$

where $C_{0}=e^{\frac{r}{T} \ln \left(1+\frac{1}{C}\right)}$ and $\gamma=\frac{\ln \left(1+\frac{1}{C}\right)}{T}$, and the exponential decay follows.

\section{ACKNOWLEDGEMENT}

The authors would like to thank the anonymous referee for a careful reading of the manuscript and for constructive suggestions which resulted in the present version of the paper.

\section{REFERENCES}

[BAR-LE-RAU] C. Bardos, G. Lebeau and J. Rauch, Sharp sufficient conditions for the observation, control, and stabilization of waves from the boundary. SIAM J. Control Optim. 30 (1992), no. 5, 1024-1065. MR1178650(94b:93067)

[BEL] M. Bellassoued, Decay of solutions of the elastic wave equation with a localized dissipation, Annales de la Faculté des Sciences de Toulouse, XII (3), 2003, 267-301. MR2030088 (2005b:35169)

[CA-OQ] M. M. Cavalcanti and H. P. Oquendo, Frictional versus viscoelastic damping in a semilinear wave equation. SIAM J. Control Optim. 42 (2003), no. 4, 1310-1324. MR2044797(2005d:35257) 
[CA-DO-FU-SO] M. M. Cavalcanti, V. N. Domingos Cavalcanti, R. Fukuoka and J. A. Soriano, Uniform stabilization of the wave equation on compact surfaces and locally distributed damping, Methods Appl. Anal. 15(4) (2008), 405-426. MR2550070 (2010m:35285)

[CA-DO-FU-SO-1] M. M. Cavalcanti, V. N. Domingos Cavalcanti, R. Fukuoka and J. A. Soriano, Uniform stabilization of the wave equation on compact surfaces and locally distributed damping, Transactions of AMS 361(9) (2009), 4561-4580. MR2506419(2010e:58030)

[CA-DO-FU-SO-2] M. M. Cavalcanti, V. N. Domingos Cavalcanti, R. Fukuoka and J. A. Soriano, Asymptotic stability of the wave equation on compact manifolds and locally distributed damping: a sharp result. Arch. Ration. Mech. Anal. 197 (2010), no. 3, 925-964. MR2679361 (2012a:35331)

[CHR] H. Christianson, Semiclassical non-concentration near hyperbolic orbits, J. Funct. Anal. 246 (2007), no. 2, 145-195. MR2321040 (2008k:58058)

[DA] C. M. Dafermos, Asymptotic behavior of solutions of evolution equations. Nonlinear evolution equations (Proc. Sympos., Univ. Wisconsin, Madison, Wis., 1977), pp. 103-123, Publ. Math. Res. Center Univ. Wisconsin, 40, Academic Press, New York-London, 1978. MR513814 (80i:35019)

[DA-LA-TO] M. Daoulatli, I. Lasiecka and D. Toundykov, Uniform energy decay for a wave equation with partialy supported nonlinear boundary dissipation without growth restrictions, DCDS-S, 2, 1 (2009). MR2481581(2010f:35227)

[DEH-LE-ZUA] B. Dehman, G. Lebeau and E. Zuazua, Stabilization and control for the subcritical semilinear wave equation. Anna. Sci. Ec. Norm. Super. 36, 525-551 (2003). MR2013925 (2004i:35223)

[HI] M. Hitrik, Expansions and eigenfrequencies for damped wave equations, Journées équations aux Dérivées Partielles (Plestin-les-Grèves, 2001), Exp. No. VI, 10 pp., Univ. Nantes, Nantes, 2001. MR.1843407(2002h:58050) P. Gerárd, Microlocal defect measures, Com. Par. Diff. Eq. 16 (1991), 17611794. MR:1135919 (92k:35027)

[GES-MES] A. Guesmia and S. A. Messaoudi, A general decay result for a viscoelastic equation in the presence of past and finite history memories, Nonlinear Analysis: Real World Applications 13 (2012), 476-485. MR2846857 (2012i:35213)

[MAR]

[RI-SA] J. E. Muñoz Rivera and A. Peres Salvatierra, Asymptotic behaviour of the energy in partially viscoelastic materials. Quart. Appl. Math. 59 (2001), no. 3, 557-578. MR 1848535 (2002f:35038)

[NA] M. Nakao, Decay and global existence for nonlinear wave equations with localized dissipations in general exterior domains. New trends in the theory of hyperbolic equations, 213-299, Oper. Theory Adv. Appl., 159, Birkhäuser, Basel (2005). MR2175918 (2006i:35250)

[NA1] M. Nakao, Energy decay for the wave equation with boundary and localized dissipations in exterior domains, Math. Nachr. 278(7-8) (2005), 771-783. MR2141956 (2006a:35185)

[RAU-TAY] J. Rauch and M. Taylor, Decay of solutions to nondissipative hyperbolic systems on compact manifolds. Comm. Pure Appl. Math. 28(4) (1975), 501-523. MR.0397184 (53:1044a)

[QIN] T. Qin, Asymptotic behavior of a class of abstract semilinear integrodifferential equations and applications. J. Math. Anal. Appl. 233 (1999), no. 1, 130-147. MR1684377 (2000a:45023) 
[TOUN] D. Toundykov, Optimal decay rates for solutions of nonlinear wave equation with localized nonlinear dissipation of unrestricted growth and critical exponents source terms under mixed boundary, Nonlinear Analysis T. M. A. 67(2) (2007), 512-544. MR2317185 (2008f:35257)

[TRI-YAO] R. Triggiani and P. F. Yao, Carleman estimates with no lower-order terms for general Riemannian wave equations. Global uniqueness and observability in one shot, Appl. Math. and Optim. 46 (Sept./Dec. 2002), 331-375. Special issue dedicated to J. L. Lions. MR.1944764(2003j:93042)

[ZUA] E. Zuazua, Exponential decay for the semilinear wave equation with locally distributed damping. Comm. Partial Differential Equations 15 (1990), no. 2, 205-235. MR 1032629 (91b:35076)

Department of Mathematics, State University of Maringá, 87020-900, Maringá, PR, BRAZIL

E-mail address: mmcavalcanti@uem.br

Department of Mathematics, State University of Maringá, 87020-900, Maringá, PR, BRAZIL

E-mail address: vndcavalcanti@uem.br

Department of Mathematics, State University of Ceará-FAFidAM, 62930-000, Limoeiro do Norte, CE, BraziL

E-mail address: flavio.falcao@uece.br 